\title{
Research on supply and demand matching of taxi in the Internet+ Era
}

\author{
Fengli Li
}

\author{
North China Electric Power University,Huadian-Lu 689,071000 Baoding,China \\ 18561516152@163.com
}

Keywords: Multi-index evaluation model, Taxi supply and demand ,TOPSIS method

\begin{abstract}
In order to study the taxi resource supply and demand matching in the Internet+ era,and provide advice on taxi resource allocation, we do research from two aspects--Firstly in space,we build multi-index evaluation model with TOPSIS $^{[1]}$ (Technique for Order Preference by Similarity to an Ideal Solution)method.Secondly,from the angle of time,we calculate no-load rate and mileage utilization ratio to reflect on the level of taxi service.According to analyzing comprehensive evaluation index, we get:In different cities, the degrees of matching are intervallic,mainly due to the urban economic development;besides.In the same area,the matching degree is positively correlated with the morning peak and evening peak.
\end{abstract}

\section{Introduction}

We are discussing the problem basing on the problem B in CUMCM 2015.

Through observations of date recorded on the platform"Intelligent Travel Platform of Kuaidi\&Didi Group " ${ }^{[2]}$,we get: there is no similarity among different cities,and in one city,the taxi resource supply and demand matching varies violently during morning and evening periods.

Considering from two aspects.

One is from the angle of space,we choose mileage utilization ratio ${ }^{[3]}$, average passenger waiting time $^{[3]}$,vehicle load ratio and vehicle ownership ${ }^{[3]}$ as evaluation index.After data preprocessing,non dimensional,data normalization, under any index, the attribute value is bigger,the performance is better.So we build the multi-index evaluation with TOPSIS method.The figure of comprehensive evaluation index is proportional to the level of the taxi service.

The other one is from the angle of time,we record the number of available taxis and demand of taxis in certain areas of Guangzhou per hour.Based on the average value of the recorded data,we can calculate no-load rate and mileage utilization ratio,which can stand for the supply and demand of taxi,and reflect on the level of taxi service.

\section{Creating and solving multi index evaluation model}

\subsection{Creating and solving multi index evaluation model of sapce}

Step 1 list calculating formula of the evaluation index

mileage utilization ratio=business mileage $(\mathrm{km}) /$ driving mileage $(\mathrm{km}) \times 100 \%$

average passenger waiting time $=$ no-load mileage $(\mathrm{km}) /$ no-load average $\operatorname{speed}(\mathrm{km} / \mathrm{h})(2)$

vehicle load ratio $=$ the numbei of vehicle load/total number of vehicle $\times 100 \%$

vehicle ownership=the numbei of vehicle/total population(million)

Step 2 normalization of attribute values

vehicle ownership $d_{i j}$ and the rest are not the same class.It dose not belong to benefit attribute or cost attribute, $b_{i j}$ is formed by the transformation of the interval.set the optimal attribute interval $\left[B_{1}, B_{2}\right]$.

$$
\begin{aligned}
& \text { When } A_{1} \leq d_{i j}<B_{1}, b_{i j}=1-\left(B_{1}-d_{i j}\right) /\left(B_{1}-A_{1}\right) \\
& \text { when } B_{1} \leq d_{i j} \leq B_{2}, b_{i j}=1 \\
& \text { when } B_{2}<d_{i j} \leq A_{2}, b_{i j}=1-\left(d_{i j}-B_{2}\right) /\left(A_{2}-B_{2}\right) .
\end{aligned}
$$




\section{Otherwise, $b_{i j}=0$}

Step 3 standardization of attribute values

The standardization can eliminate the influence of dimension, and making each variable has the same performance.After standardization of vector,in any city,the sum of the squares of the attribute values of the same index is equal to one,

$$
\begin{gathered}
a_{i j}=\frac{b_{i j}-\overline{b_{j}}}{\sqrt{\frac{\sum_{i=1}^{4}\left(b_{i j}-\frac{\sum_{i=1}^{4} b_{i j}}{4}\right)^{2}}{3}}} \\
g_{i j}=a_{i j} / \sqrt{\sum_{i=1}^{4} a_{i j}^{2}}
\end{gathered}
$$

Step 4 determine positive ideal solution $C_{j}^{+}$and negative ideal solution $C_{j}^{-}$

Assuming weight vector $w$, then $C_{i j}=w_{j} * g_{i j}$.

when ${ }^{j}$ belongs to benefit attribute, ${ }^{c_{j}^{+}}=\max c_{i j},{ }^{-}{ }_{j}^{-}=\min c_{i j}$.

when ${ }^{j}$ belongs to cost attribute, ${ }^{+}{ }_{j}^{+}=\max c_{i j}, c_{j}^{-}=\min c_{i j}$.

Step 5 calculate comprehensive evaluation index $f_{i}$

The figure of $f_{i}$ is proportional to the level of the taxi service.

$$
\begin{gathered}
s_{i}^{+}=\sqrt{\sum_{j=1}^{4}\left(c_{i j}-c_{j}^{+}\right)^{2}} \quad s_{i}^{-}=\sqrt{\sum_{j=1}^{4}\left(c_{i j}-c_{j}^{-}\right)^{2}} \\
f_{i}=s_{i}^{-} /\left(s_{i}^{-}+s_{i}^{+}\right)
\end{gathered}
$$

Assuming weight vector $w=[0.4,0.2,0.3,0.1]^{T}$.

From the above equation,we can get the specific date shown in Table 1:

Table 1 The figure of comprehensive evaluation index of four cities

\begin{tabular}{llllll}
\hline \hline & $c_{j}^{+}$ & $c_{j}^{-}$ & $f_{i}$ & & ranking \\
Beijing & 0.3285 & -0.2125 & 0.4341 & 4 & \\
Wuhan & 0.1015 & -0.1354 & 0.5299 & 1 & \\
Guangzhou & 0.1581 & -0.247 & 0.4745 & 3 & \\
Yinchuan & -0.08 & 0.055 & 0.4888 & 2 & \\
\hline \hline
\end{tabular}

\subsection{Creating and solving multi index evaluation model of time}

We record the data of available taxis and waiting passengers.Put data into following (1) (5) to get no-load rate ,combined withmileage utilization ratio per hour in Guangzhou. Do corresponding line-chart.We can derived to obtain the impact of morning and evening peak.

mileage utilization ratio=business mileage $(\mathrm{km}) /$ driving mileage $(\mathrm{km}) \times 100 \%$

no-load rate $=($ available taxis-waiting passengers $) /$ available taxis $\times 100 \%$

\section{Analyzing multi index evaluation model}

\subsection{Analyzing multi index evaluation model of space}

According to analyzing comprehensive evaluation index $f_{i}$, we draw a conclusion:with the figure of comprehensive evaluation index $f_{i}$ increasing, the taxi resources supply can meet demand better,the level of taxi service is higher. We can learn that the ranking of matching degree of supply 
and demand from high to low is:Wuhan, Yinchuan,Guangzhou,Beijing.

The matching degree is mainly determined by following three factors:urban economic development,population quantity,traffic flow.

In the first line cities,like Beijing,Guangzhou,there are rich taxi resources.But the population quantity cause huge demand,which limits the matching degree of the supply and demand.

In Yinchuan and other three or four line cities, the number of available taxis is smaller,at the same time, the lower living standards reduce demand,which can explain why comprehensive evaluation index $f_{i}$ is relatively high.

As for some typical two line cities, like Wuhan,there exists a stable balance between supply and demand,the level of service is the highest.

\subsection{Analysing multi index evaluation model of time}

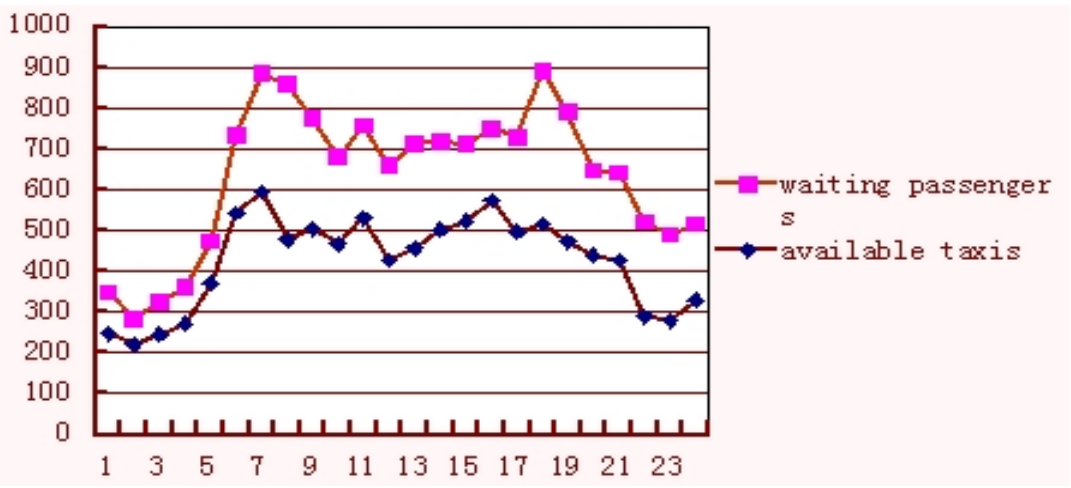

Fig 1 the number of passenger and taxi per hour in Guangzhou

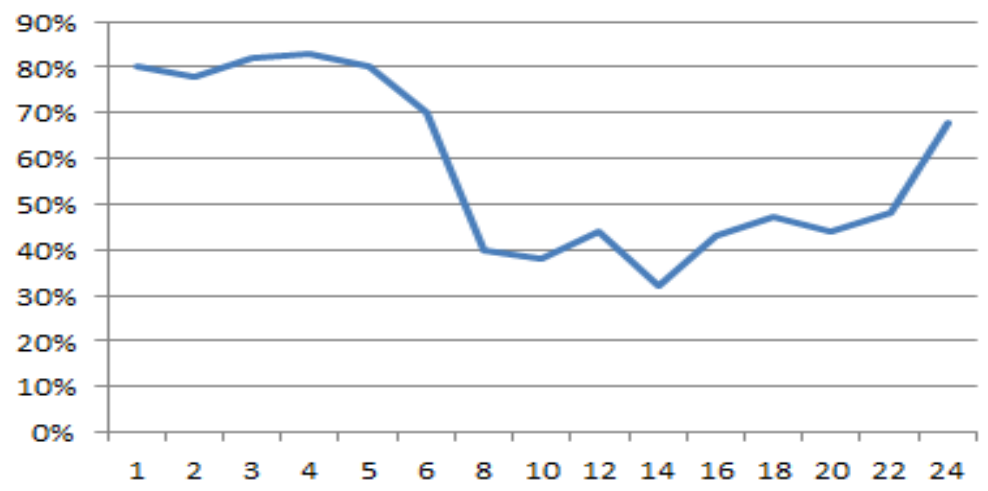

Fig 2 no-load rate per hour in Guangzhou

As shown in Figure 1 and Figure 2,the number of waiting passengers reaches its rush hour between 7:00 and 9:00, which also happen between 17:00 and19:00.Consequently, the no-load rate reduce. We can derived to obtain the matching degree of supply and demand,to evaluate the level of taxi service in a day.

\section{Summary}

The above evaluation model is built on basis of specific time and space.On the basis of the analysis above,we can draw a conclusion:the supply and demand matching is mainly influenced by the urban economic development among different cities; while in the same area, the matching degree is positively correlated with the morning and evening peak. If the model is extended to the country, it will be able to predict the situation in different time and space, which has great significance in taxi resource allocation. 


\section{References}

[1]SI Shoukui, SUN Xijing. Mathematical Modeling. National Defense Industry Press, 2011, p.345-347.

[2]information on :http://v.kuaidadi.com.

[3]Three indicators to measure the taxi supply and demand-mileage utilization ratio,vehicle Load ratio and vehicle ownership. [J]Transport manager world, 2007.05.05. 